This is a post peer-review, pre-copyedit version of:

Ferrari, F. \& Orlandelli, E, Proof-theoretic pluralism, in Synthese

First Online29 April 2019.

(C) Springer Netherlands

The final authenticated version is available online at:

https://doi.org/10.1007/s11229-019-02217-6

This version is subjected to Springer terms for reuse that can be found at:

https://www.springer.com/gb/open-access/authors-rights/self-archiving-policy/2124 


\title{
Proof-theoretic pluralism
}

\begin{abstract}
Starting from a proof-theoretic perspective, where meaning is determined by the inference rules governing logical operators, in this paper we primarily aim at developing a proof-theoretic alternative to the modeltheoretic meaning-invariant logical pluralism discussed in [1]. We will also outline how this framework can be easily extended to include a form of meaningvariant logical pluralism. In this respect, the framework developed in this paper - which we label two-level proof-theoretic pluralism - is much broader in scope than the one discussed in [1]
\end{abstract}

\section{Introduction}

Logical pluralism is the thesis that there is a plurality of admissible logics. This idea has been developed in a variety of ways. Two versions of it have been particularly influential in recent discussions: a broadly Carnapian version of meaning-variant logical pluralism (MVLP, for short) wherein the meaning of the logical operators varies from one admissible logic to the other, and a meaning-invariant one (MILP), wherein the meaning of the logical operators remains constant among the various admissible logics. Since the publication of [1, much of the focus has been on this second, meaning-invariant, version of logical pluralism. In [1, Beall and Restall develop a model-theoretic framework for MILP where all of classical, intuitionistic, and relevant logics are admissible.

Starting from a proof-theoretic perspective, where meaning is determined by the inference rules governing logical operators, this paper will be mostly devoted to developing a proof-theoretic alternative to the model-theoretic MILP discussed in [1]. After completion of this task, we will briefly outline how this

Final draft. See here for the published version.

Address(es) of author(s) should be given 
framework can be extended to include a form of MVLP. In this respect, the general framework developed in this paper - which we label two-level prooftheoretic pluralism - is much broader in scope than the one discussed in [1: modulo a common admissibility criterion, we obtain both a variety of families of logic where the operators have a different meanings - MVLP -, as well as a variety of different logics within each family wherein the operators share the same meaning - MILP. Our main motivation for introducing the general framework of two-level proof-theoretic pluralism is to indicate a strategy to reply to one of the main criticisms moved against [1, namely that it is not pluralist enough in that it excludes a variety of logics that have been recently employed in philosophical debates - e.g. in relation to the semantic paradoxes. We take that the ability to include such logics within our two-level proof theoretic pluralism gives a significant advantage to our pluralism over the model-theoretic one offered in 1 .

The proof-theoretic version of MILP developed here starts from the assumption that the meaning of each logical operator is completely determined by its left and right introduction rules in sequent calculi. We then provide an admissibility criterion for a logic based on Belnap's notion of harmony [3] and we show that all of classical, intuitionistic, dual-intuitionistic, and some relevance logics are admissible. This gives us a form of MILP because the calculi for these different logics, while differing at the structural level, are based on the same set of operational rules. In developing the framework we will also show how our proposal fully addresses the criticisms moved by Teresa Kouri [19] to a version of proof-theoretic MILP presented by Greg Restall [30. According to Kouri, Restall's proposal cannot allow for any relevance logics as admissible while at the same time avoiding the charge of meaning-variance. By adopting Belnalp's notion of harmony as a criterion of admissibility, some relevance logics are admissible in our framework without having to change the operational rules and thus the meaning of logical operators. With this criterion of admissibility in hand, we will show that all of the harmonious logics in this meaning-invariant family of admissible logics meet the three features of Necessity, Formality, and Normativity discussed in 11. Instead of taking these features as constitutive of logic - as it is done in [1] - we take them to be pre-theoretical desiderata the satisfaction of which provides us with abductive evidence for the material adequacy of our proposal.

The paper is structured as follows. Section 2 introduces the basic framework of proof-theoretic MILP and Section 3 shows how classical and intuitionistic logics can coexist within the basic framework. Section 4 presents a problem for the basic framework that has been raised in [19, namely that it is not clear whether the basic framework can be supplemented with an admissiblity criterion that includes not only classical and intuitionistic logics but also some relevance logic. Then, in Section 5 we will argue that Belnap's proof-theoretic notion of harmony provides such a criterion and in Section 6 we will show how relevance logics behave in an harmony-based proof-theoretic pluralism. Section 7 develops our proposal in more detail by arguing that it gives us a two-level proof-theoretic pluralism that admits of both a form of MILP and a 
Table 1 Operational rules

\begin{tabular}{ll}
\hline$\left.\frac{\Gamma \vdash \Delta, A}{\neg A, \Gamma \vdash \Delta} L\right\urcorner$ & $\frac{A, \Gamma \vdash \Delta}{\Gamma \vdash \Delta, \neg A} R \neg$ \\
$\frac{A, \Gamma \vdash \Delta \quad B, \Gamma \vdash \Delta}{A \vee B, \Gamma \vdash \Delta} L \vee$ & $\frac{\Gamma \vdash \Delta, A_{i}}{\Gamma \vdash \Delta, A_{1} \vee A_{2}} R \vee_{i}, i \in\{1,2\}$ \\
$\frac{A_{i}, \Gamma \vdash \Delta}{A_{1} \wedge A_{2}, \Gamma \vdash \Delta} L_{i}, i \in\{1,2\}$ & $\frac{\Gamma \vdash \Delta, A \quad \Gamma \vdash \Delta, B}{\Gamma \vdash \Delta, A \wedge B} R \wedge$ \\
$\frac{\Pi \vdash \Sigma, A \quad B, \Gamma \vdash \Delta}{A \supset B, \Pi, \Gamma \vdash \Delta, \Sigma} L \supset$ & $\frac{A, \Gamma \vdash \Delta, B}{\Gamma \vdash \Delta, A \supset B} R \supset$ \\
$\frac{A[z / x], \Gamma \vdash \Delta}{\exists x A, \Gamma \vdash \Delta} L \exists(z$ fresh $)$ & $\frac{\Gamma \vdash \Delta, A[y / x]}{\Gamma \vdash \Delta, \exists x A} R \exists$ \\
$\frac{A[y / x], \Gamma \vdash \Delta}{\forall x A, \Gamma \vdash \Delta} L \forall$ & $\frac{\Gamma \vdash \Delta, A[z / x]}{\Gamma \vdash \Delta, \forall x A} R \forall(z$ fresh $)$ \\
\hline
\end{tabular}

form of MVLP. In Sections 8, 9, and 10 we discuss the features of Necessity, Formality, and Normativity within our proof-theoretic MILP.

\section{The basic framework of proof-theoretic pluralism}

In this section we are going to introduce what we call the basic framework of proof-theoretic MILP. We call it the basic framework because it presents only some core philosophical ideas and the technical framework of sequent calculi that we share with other proponents of proof-theoretic MILP - e.g., with minor variations the basic framework is adopted, among others, by Dicher [6], Došen 9], Hjortland [18], Kouri [19, Paoli [25, and Restall [30] 11

Let us start by introducing sequent calculi. As standard, we define sequent calculi by giving a set of operational rules for the operators of the object language and by giving a set of structural elements that is composed by a set of structural rules and by a set of structural information regarding how

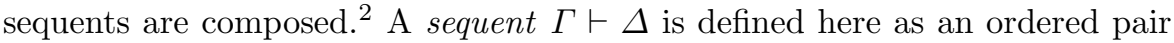
of multisets - i.e., sets with repetition or disordered sequences - of formulas of a given language ${ }^{3}$ The operational and structural rules of inference that we will consider are given, respectively, in Tables 1 and $2^{4}$

The main idea of proof-theoretic MILP is that the meaning of each logical operator is completely determined by its left and right operational rules, and

1 These proposals differ mostly at the technical level and, consequently, in the set of logics considered. The basic framework we are going to consider is a variation of the one in 1930 .

2 As usual, a rule is called structural if it doesn't involve essentially any operator of the object language, else it is called operational.

3 The main alternatives being to define sequents as pairs of either sets or sequences of formulas, or as $n$-tuples of sets/multisets/sequences of formulas as in 18 .

4 For the sake of easiness, we limit ourselves to a first-order language without identity. 
Table 2 Structural rules

\begin{tabular}{ll}
\hline$\overline{A \vdash A}^{I d}$ & $\frac{\Pi \vdash \Sigma, A}{\Pi, \Gamma \vdash \Delta, \Gamma \vdash \Delta} \mathrm{Cut}$ \\
$\frac{\Gamma \vdash \Delta}{A, \Gamma \vdash \Delta}{ }_{L-W k n}$ & $\frac{\Gamma \vdash \Delta}{\Gamma \vdash \Delta, A} R-W k n$ \\
$\frac{A, A, \Gamma \vdash \Delta}{A, \Gamma \vdash \Delta}{ }_{L-C t r}$ & $\frac{\Gamma \vdash \Delta, A, A}{\Gamma \vdash \Delta, A}{ }_{R-C t r}$
\end{tabular}

that different logics can be obtained by changing the structural elements of deduction while maintaining the same set of operational rules. In particular, a family of logics is obtained by having the operational rules given in Table 1 and by changing the dynamic structural elements of deduction that are given by the structural rules of inferences given in Table 2 and/or by changing the static structural elements of deduction that are given by the definition of how sequents are composed ${ }^{5}$

Following Restall [30, we consider three different logics that can be obtained by changing the static elements of deduction - i.e., the definition of how sequents $\Gamma \vdash \Delta$ are composed, where in general $\Gamma$ and $\Delta$ are multisets of formulas:

1. if neither $\Gamma$ nor $\Delta$ is restricted we obtain classical logic;

2. if $\Delta$ can contain at most one formula and $\Gamma$ is unrestricted, we obtain intuitionistic logic;

3. if $\Gamma$ can contain at most one formula and $\Delta$ is unrestricted, we obtain dual-intuitionistic logic.

Cases 1 and 2 of the definition of sequents give, respectively, Gentzen's [14] calculus for classical logic LK and Gentzen's calculus for intuitionistic logic LJ. Finally, case 3 gives Urbas' [40] calculus for dual-intuitionistic logic LDJ ${ }^{6}$

We thus obtain a proof-theoretic form of logical pluralism which encompasses classical, intuitionistic and dual-intuitionistic logics. Moreover, we have assumed that the meaning of a logical operator is given by the operational rules governing its formal behavior, and these different logics have been obtained by changing the structure of sequents while maintaining the same set of operational rules. In this way, with proof-theoretic pluralism 'we have one language, and three logical consequence relations on that language' [30, p. 284].

To sum up, the version of proof-theoretic MILP outlined so far is able to neatly avoid the charge that the meaning of some logical operator varies across the different admissible logics. We take this to be a welcome result.

\footnotetext{
5 Static and dynamic structural elements of deduction are closely related: a change in one of the two might impose some change in the other - e.g., if sequents are defined as pairs (or $n$-tuples) of sets, and not of multisets (or of ordered lists), then the structural rules of contraction get out of the picture.

6 To be precise, we have a version of Urbas' logic with a sub-optimal implication. To get full dual-intuitionistic logic we would have to change the rules for implication in such a way that we would get a sub-optimal intuitionistic implication, cf. [40, p. 442].
} 


\section{Classical and intuitionistic logic within the basic framework}

Before moving on to developing the basic framework into a full proposal, we would like to pause for a minute on the task of illustrating how classical and intuitionistic logic can coexist in approaches based on the basic framework. This allows us both to exemplify the sort of pluralism we are proposing and to dispel some worries about our appeal to both single- and multiple- conclusion calculi.

As we have said, LJ and $\mathbf{L K}$ share the same set of operational rules, the only difference between them lying at the structural level. As a consequence of this difference, in $\mathbf{L J}$ all instances of operational rules will have at most one formula in the right-hand side of their sequents whereas in LK they can contain more than one formula. But this does not mean that they are instances of different operational rules: they are different instances of one and the same rule. In particular, all $\mathbf{L J}$-instances of one operational rule are also $\mathbf{L K}$-instances of the same rule, but not vice versa. Thus, some classical theorems - such as Peirce's law: $((A \rightarrow B) \rightarrow A) \rightarrow A$ - won't be theorems of intuitionistic logic. But this does not affect the meaning of implication since, as it is usual in proof-theoretic semantics, we assume a molecularist theory of meaning: the meaning of an operator is completely determined by its operational rules and not by the set of theorems where it occurs. The structural restriction needed to move from $\mathbf{L K}$ to $\mathbf{L J}$

involves no essential reference to any connectives, [so] it is hard to see how it could be explicable as arising from divergence of meaning of connectives [17, p. 10].

Thus, we can maintain that in switching from classical to intuitionistic logic we are not changing the meaning of the logical operators: LJ and LK share the same meaning-conferring operational rules. They differ only at the structural level in that one is a single-conclusion calculus and the other is a multipleconclusion one.

Some proponents of proof-theoretic semantics [11,36] argue that multipleconclusion calculi cannot be meaning-conferring as we have taken them to be. Nevertheless, others [9, 28, 29, argue that multiple-conclusion calculi can be meaning-conferring and there are many different explications of multiple conclusions - e.g., Došen [9] proposes that they are enthymematic and Restall 29] proposes a bilateralist reading of them. This is not the place to enter into the single vs. multiple conclusions debate. We will simply assume that some explication of multiple-conclusion calculi is correct and that both single- and multiple-conclusion calculi can be meaning-conferring.

Even if both single- and multiple-conclusion calculi can be meaning conferring, someone might still object that we have to endorse either multipleconclusion calculi or single-conclusion calculi across the board $]^{7}$ Instead, we propose that we can endorse both kinds of calculi and that, even if in general

\footnotetext{
7 Thanks are due to an anonymous referee for pressing us on this point.
} 
we can use multiple-conclusion calculi, we have to resort to single-conclusion ones in situations where we have to reason constructively. The need to restrict ourselves to single-conclusion calculi - such as $\mathbf{L J}$ - when reasoning constructively is well motivated since, as shown by the following argument by Neil Tennant, we cannot reason constructively with multiple conclusions.

In general [a multiple-conclusion calculus] smuggles in non-constructivity from the back door.[..] If one is told that $\Gamma \vdash A_{1}, \ldots, A_{n}$ is valid in the extended sense for multiple-conclusion arguments just in case $\Gamma \vdash A_{1} \vee \ldots \vee A_{n}$ is valid in the usual sense for single-conclusion arguments, the intuitionist can demand to know precisely which disjunct $A_{i}$, then, proves to be derivable form $\Gamma$. No answer to such a question can be provided in general with the multiple-conclusion calculus of the classical logician. 39, p. 320; notation changed]

This argument shows that we must use single-conclusion calculi when we are reasoning constructively.

Three objections to Tennant's argument have been raised by Florian Steinberger 34, but, as we are now going to show, none of them works in the present context. Two objections are that single-conclusion calculi are not necessary nor, respectively, sufficient for constructivity. That they are not necessary is shown by the existence of a multiple-conclusion calculus G3im for intuitionistic logic. That they are not sufficient is shown by the existence of a single conclusion calculus for classical logic $\mathbf{G} 3 \mathbf{i}+G e m-a t .8$ However, G3im is obtained by changing the operational rules for implication and for the quantifiers with respect to the calculus G3c for classical logic, and $\mathbf{G} 3 \mathbf{i}+G e m$-at is obtained by extending the calculus G3i for intuitionisitc logic with a rule where negation occurs essentially. Both calculi are obtained by changing the meaning of some logical operator with respect to the original calculus. If we want to avoid the charge of meaning-variance, we cannot appeal to these calculi. Hence, given that we want to avoid meaning-variance, we can safely conclude that single conclusions are necessary and sufficient for constructivity ${ }^{9}$

The other objection relies on Tennant's aim of showing that a logical monist has to choose single-conclusion calculi and therefore to adopt a logic not stronger than intuitionistic logic. But, as Steinberger rightly claims, the appeal to constructivity in an argument against multiple-conclusion calculi, and thus in favour of constructive logics, is circular. This objection does not affect our use of Tennant's argument: we have used it to argue that we should use single-conclusion calculi whenever we are in a constructive context, and not to ban non-constructive logics. Hence, no circularity is involved.

Thus, none of Steinberger's objections can be raised against our use of Tennant's argument for showing that when we have to reason constructively we should use a single-conclusion calculus such as LJ. We conclude that the choice of whether using a single- or a multiple-conclusion calculus depends

\footnotetext{
8 See [24 pp. 108-121] for the details of these two calculi.

9 To the best of our knowledge, all potential counterexamples to the necessity and sufficiency of single-conclusion calculi for constructivism involve meaning-variance.
} 
on whether we have to reason constructively or not. Because we are working within a pluralist framework there is no obstacle in accepting that sometimes we may have to reason constructively and some other times we are free to reason non-constructively. Hence, we find it natural that both single- and multiple-conclusion calculi should be taken to be meaning-conferring.

The choice of whether reasoning according to $\mathbf{L J}$ or $\mathbf{L K}$, and therefore whether using single or multiple conclusions, depends on the context - in this respect, part of our motivations for going pluralist are closely related to those at the core of Stewart Shapiro's proposal elaborated in [33. In a paradigmatic classical context, such as when we are reasoning within classical real analysis, we can safely use the multiple-conclusion calculus LK. By constrast within intuitionistic analysis we cannot allow for multiple conclusions and we have to resort to a single-conclusion calculus such as $\mathbf{L J}$. To illustrate, we consider a basic theorem of classical real analysis such as the intermediate value theorem:

(IVT) Let $f:[a, b] \longrightarrow \mathbb{R}$ be a continuous function such that $f(a)<0<f(b)$; then there is $c \in[a, b]$ such that $f(c)=0$.

In intuitionistic analysis IVT does not hold. The problem is, roughly, that the two standard proofs of IVT in classical analysis - the first being based on suprema and the second being based on an interval-halving argument do not work in constructive settings. The reason is that while these proofs show the existence of such a $c$, they do not provide an algorithm outputting this $c$. These are non-constructive proofs that don't go through when we are reasoning with a single-conclusion calculus such as $\mathbf{L J}$.

This discussion leads us to consider an important question: whether each logic within our pluralist framework is universally applicable - i.e., whether it holds unrestrictedly in all contexts. As it is well known, intuitionistic analysis, being based on Brouwer's choice sequences, is inconsistent with classical logic. This problem can be solved in two opposite ways: either we reject intuitionistic analysis or we restrict the universal applicability of logic. On the one hand, Beall and Restall [1, pp. 118-120] keeps the universal applicability of logics by rejecting choice sequences - and thus by rejecting intuitionistic analysis - and by claiming that we can accept only those branches of constructive mathematics, such as Bishop's constructive analysis, that are consistent with classical logic. On the other hand, Shapiro [33, pp. 70-72] restricts the universal applicability of logic so that we can keep choice sequences - and thus intuitionistic analysis - and by saying that a logic cannot be applied whenever it would yield to inconsistency. In this paper we won't dig deeper on this intricate issue. We just would like to point out that within the pluralist framework developed here the most natural choice is to side with Shapiro by suitably restricting the universal applicability of logic. This option is not forced upon us since it is possible, albeit less natural, to keep the universal applicability of logic while rejecting some commonly used, and otherwise well-behaved, mathematical theories such as intuitionistic analysis. 


\section{Relevance and Restall's framework}

Having shown that within the basic framework it is possible to have both classical and intuitionistic logic without having to change the meaning of logical operators, we now move to a problem that has been raised in 19. for Restall's version of the basic framework as developed in [30]: its incompatibility with relevance logics.

Let us call a relevance logic any logic where neither of the following paradoxes of material implications are theorems:

$$
A \supset(B \supset A) \quad \neg A \supset(A \supset B)
$$

nor are theorems the following paradoxes of strict implication ${ }^{10}$

$$
\perp \supset A \quad A \supset \top
$$

As argued in 19, Restall, who is one of the main proponents of the basic framework, should want to extend his pluralism to some relevance logic for at least two different reasons. First, a relevance logic is among those considered in Beall and Restall's [1] model-theoretic MILP. Second, a pluralism which excludes all relevance logics is in danger of being too narrow and thus rather uninteresting. As a further reason, Restall [30] uses considerations about relevance to motivate his adoption of logical pluralism, and therefore a form of logical pluralism that is incompatible with relevance logics doesn't seem to be what he is looking for.

It seems easy to extend the basic framework - as well as Restall's proposal - to include some relevance logics. We have just to start from any one of the three cases of the definition of sequents, let's say with sequents $\Gamma \vdash \Delta$ where neither $\Gamma$ nor $\Delta$ are restricted, and remove the rules of weakening ( $L-W k n$ and $R-W k n)$ from the set of structural rules given in Table 2. This is sufficient to obtain a relevance logic according to the definition of relevance given above ${ }^{11}$ To wit, the left and right rules of weakening are needed to derive the paradoxes of material implication as in the following derivations:

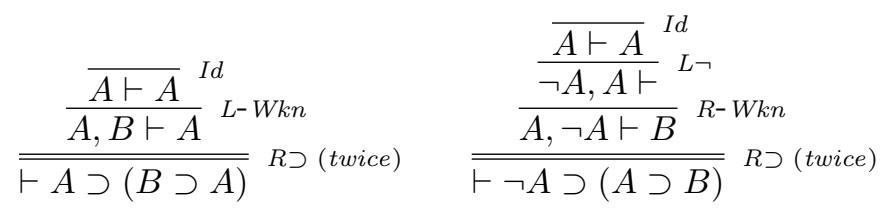

and the same holds for the paradoxes of strict implication:

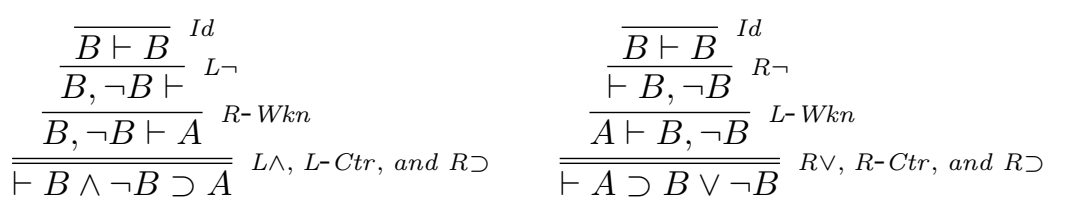

10 Where $\perp$ stands for some contradiction and $T$ for some theorem.

11 Notice that in these calculi it is hard to give a completely satisfactory account of restricted quantification in the sense of [2]. E.g, if all Fs are Gs is formalised as $\forall x(F x \supset G x)$, the inference from all $F$ s are $G$ s and $F a$ to $G a$ is valid, but that from everything is $a$ to all Fs are Gs is not valid. This issue is beyond the scope of this paper. 
However, we still have to check whether the calculus obtained by removing the rules of weakening from $\mathbf{L K}$ - let us call it $\mathbf{L K R}$ - gives us something that we might properly call a logic in Restall's framework. As Kouri [19] points out, Restall [30] has provided no correctness-criterion for logics. However, such a criterion is needed since without it we would have to accept as logics some calculi that we should want to rule out - e.g., a calculus where sequents $\Gamma \vdash \Delta$ are such that either one or both of $\Gamma$ and $\Delta$ must be empty.

Kouri argues that the natural way to provide such criterion within Restall's framework is by claiming that a calculus gives us an admissible logic if and only if its valid - i.e., derivable - sequents are compatible with Restall's reading of sequents; where, according to Restall, a sequent $\Gamma \vdash \Delta$ is read as a claim about logical consequence:

$(\star)$ every evaluation which makes all the formulas in $\Gamma$ true makes some formula in $\Delta$ true.

Thus we have to rule out all calculi whose sequents can't be read as 'every evaluation which makes all the formulas in $\Gamma$ true makes some formula in $\Delta$ true', and this immediately rules out LKR. The problem is that the rules of weakening are indeed valid under Restall's reading of sequents: if every evaluation which makes all formulas in $\Gamma$ true makes some formula in $\Delta$ true, then also every evaluation which makes true all formulas in $\Gamma, A$ makes some formula in $\Delta, B$ true. This argument shows that if we add to a valid sequent $\Gamma \vdash \Delta$ a formula either to the left or to the right of $\vdash$ we obtain a valid sequent, and therefore no calculus where the rules of weakening are not valid provides an admissible logic. Hence neither LKR nor any other relevance logic is admissible according to the criterion provided above.

The only way to avoid this negative conclusion, Kouri [19] continues, would be to change the reading of sequents into the intensional one considered in 12. However, allowing this move would immediately give rise to the meaningvariance objection. In fact, in relevance logics we would have a disjunction the so-called intensional disjunction $\oplus$ (see Table 3) - that differs from the one we have in the other admissible logics, see [19, Sect. 4] for the details.

Given the argument above, Kouri concludes that these problems with relevance logics

will be common with any non- ad hoc reinterpretation of how to read the sequents that Restall uses. There will be no way to read the sequent in such a way that, classical, intuitionistic and relevance logic are included in the proof-theoretic pluralism. [19, p. 1251]

Since Restall's framework is an instance of the basic framework, it seems that Kouri's argument shows that there is no way to develop the basic framework in such a way that it has a criterion of admissibility including all of classical, intuitionistic, dual-intuitionistic, and relevance logics. 


\section{A proof-theoretic admissibility criterion}

Kouri's conclusion is, we believe, unwarranted. Kouri is certainly right in claiming that the basic framework has to be supplemented with a criterion of correctness and that if this criterion is given in terms of the compatibility of valid sequents with Restall's reading $(\star)$, then relevance logics are ruled out. But Restall's reading of sequents is model-theoretic - it is based on the model-theoretic notions of truth and evaluation - and, therefore, it gives us a model-theoretic criterion of correctness. This may come as a surprise since such a criterion fits rather oddly with the adoption of a proof-theoretic perspective where these model-theoretic notions should be replaced with proof-theoretic ones such as those of proof and inference. But, of course, this can be easily fixed by replacing both Restall's [30] model-theoretic reading of sequents and Kouri's [19] model-theoretic criterion of correctness with their respective proof-theoretic counterparts. As we will show, once we have fully adopted a proof-theoretic account of admissibility, we can have all of classical, intuitionistic, dual-intuitionistic and relevance logics as admissible while preserving the meaning of the logical operators. In this regard, we would like to emphasise that even if it were possible to amend the model-theoretic admissibility criterion $(\star)$ - thus making some relevance logic admissible - this wouldn't be fully in consonance with a proof-theoretic framework. A proof-theoretic criterion of admissibility - like the one we are about to introduce - is the natural choice for anyone endorsing such a framework.

First of all, we have to give a proof-theoretic reading of sequents. As it is well known, a sequent $\Gamma \vdash \Delta$ directly represents a derivability claim of the form: 'the cases in $\Delta$ are derivable from the assumptions in $\Gamma$ ', where cases, which are reminiscent of the division into cases that we often find in mathematical proofs, in general have to be read disjunctively ${ }^{12}$ Moreover, derivablity is a formal representation of the pre-theoretic notion of following from. Therefore, we can give a proof-theoretic reading of a sequent $\Gamma \vdash \Delta$ by reading it as:

$(\star \star)$ the cases in $\Delta$ follow from the assumptions in $\Gamma$.

By doing so, we replace the model-theoretic notions of truth and evaluation with the proof-theoretic notion of following from $\sqrt{13}$

\footnotetext{
12 The notion of 'cases' employed in the proof-theoretic reading of sequents is the intuitive, pre-theoretic, notion which is standard in mathematical reasoning - see, for instance, 24 - and as such should not be conflated with the model-theoretic notion of cases often used to refer to possible worlds, situations, or entities of the like rather than formulas (or propositions). In particular, it should not be conflated with the notion of case $x$ employed in 1.

13 Another possibility would be to adopt a form of bilateralism [29] and read $\Gamma \vdash \Delta$ as 'the non-co-deniability of the members of $\Delta$ follows from the acceptance of the members of $\Gamma^{\prime}$. We prefer the reading given in $(\star \star)$ - which is based on Gentzen's 14 denotational interpretation of sequents, cf. 24 p. 47] - as it is more neutral and it does not require the adoption of bilateralism. In any case our reading is compatible with bilateralism and we will make use of a bilateral interpretation of sequents when talking about the normativity of admissible logics in Section 10
} 
Next, we have to supplement this proof-theoretic reading with some criterion of correctness that delimits the set of admissible logics. The reading proposed in $(\star \star)$ does not by itself provide such a criterion since the intuitive notion of following from does not exclude any putative 'logic' by itself. This is an old problem for proof-theoretic semantics. Already in the sixties Prior [27] claimed that we cannot determine logical operators, and hence admissible logics, by reference only to proof-theoretic notions. This conclusion followed from the observation that otherwise we would have to accept as meaning-conferring the rules for operators, such as Prior's tonk, which trivialize transitive derivability relations. We have to find a way to check whether the rules of a calculus determine an admissible logic or not.

In proof-theoretic semantics the task of providing criteria of correctness for rules of inference goes under the label harmony. Intuitively, harmony asks for 'a certain consonance between the two aspects of the use of a given form of expression' 10, p. 397]. Different formal explications of the notion of harmony have been proposed in the literature ${ }^{14}$ In this paper we adopt Belnap's 3] explication of harmony in terms of conservativity and uniqueness ${ }^{15}$ Belnap's proposal starts from the observation that 'we are not defining our connectives $a b$ initio, but rather in terms of an antecedently given context of deducibility' 3. p. 131]. This is a rather natural starting point: the derivability relation is a formal explication of the notion of following from, and hence it has to satisfy the properties that we take to be distinctive of this notion. In our case, as done in 1 for the consequence relation, we start with a derivability relation $\vdash$ that is reflexive and transitive. More precisely, a derivability relation is a binary relation between multisets of fomulas that is reflexive and transitive - i.e., a set of sequents that is closed under the rules $I d$ and $C u t$. ${ }^{16}$ As a consequence, the putative proof-theoretic definition of a logical operator $\circ$ is

14 See, e.g., 7] for a sketch of some of the main options.

15 Many authors - e.g., Dummett 11, Read 28, and Steinberger 35 - criticize Belnap's definition because it is global: whether an operator is harmonious depends on the full set of rules of the calculus, and thus it might depend on the operational rules for other operators. These authors work mostly with natural deduction and present local explications of harmony that are usually based on so-called inversion principles, cf. [22]. The proof-theoretic MILP presented in this paper need an explication of harmony that is based on sequent calculi since in natural deduction the structural elements of deduction are hidden in the operational rules, but it would work also if we had used a local explication of harmony in sequent calculi instead of Belnap's global one. To illustrate, one local notion of harmony in sequent calculi is the one considered in 15, where, starting from a reflexive and transitive derivability relation, an operator is harmonious if either its right rule(s) is(are) deductively equivalent to the inverse of its left one(s) or its left rule(s) is(are) deductively equivalent to the inverse of its right one(s). Mutatis mutandis, the calculi considered here are harmonious with respect to this local explication of harmony. We have chosen Belnap's definition because it is well known and it allows us to make our point without having to dwell on many formal details; cf. 7 for an argument in favour of Belnap-like global harmony.

16 This is not the place to enter into the interesting discussion concerning whether there is a set of invariant features at the core of the concept of following from (and hence of the derivability relation). In general, we are quite liberal with respects to what are the distinctive properties of the derivability relation. In Section 7 we will argue that, with the possible exception of reflexivity, the set of distinctive properties of the derivability relation can be freely modified to obtain different families of admissible logics. 
successful if and only if the rules governing its behaviour are conservative i.e., they don't alter the derivabilty relation for the o-free language - and they satisfy uniqueness - i.e., if $\bullet$ is a notational variant of $\circ$ we must be able to show that $\circ$ and $\bullet$ are interderivable. The need for conservativity springs from the assumption that the o-free derivability relation already provides 'all universally valid deducibility-statement not involving [o]' 33, p. 132]. Uniqueness 'corresponds to a sort of principle of identity of indiscernibles' [23, p. 146] for proof-theoretic approaches to logic(s).

We have proposed to use Belnap's explication of harmony as a criterion of correctness for admissible logics in proof-theoretic pluralism. Now we have to show that this criterion marks as admissible all of classical, intuitionistic, dual-intuitionistic, as well as some relevance logics. First of all, in each one of $\mathbf{L K}, \mathbf{L J}, \mathbf{L D J}$, and $\mathbf{L K R}$, we can easily prove the uniqueness of the logical operators. For example, if $\rightarrow$ is governed by copies of the rules for $\supset$ and $\bigvee$ by copies of the rules for $\forall$ given in Table 1 , we can prove the uniqueness of implication and of the universal quantifier (in all calculi considered) as follows 17

$$
\begin{gathered}
{\frac{\overline{A \vdash A}^{I d} \quad \overline{B \vdash B}^{I d}}{A \supset B, A \vdash B}}_{L \supset}{ }^{R \supset}
\end{gathered}
$$

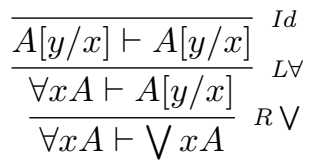

and

${\frac{\overline{A \vdash A}^{I d}}{A \rightarrow B, A \vdash B}}^{I d}{ }_{L \rightarrow} \rightarrow$
$\frac{A \rightarrow B \vdash A \supset B}{A \rightarrow B}$

and



Next, we have to prove conservativeness - i.e., we have to show, for each logical operator $\circ$, that no sequent where $\circ$ does not occur becomes derivable after the introduction of the operational rules for $\circ$. In the present context, conservativeness is an immediate consequence of cut-elimination since in cutfree calculi we have the sub-formula property: every formula $A$ occurring in a cut-free derivation of a sequent $\mathcal{S}$ is a sub-formula of the end-sequent $\mathcal{S}{ }^{18}$ It is immediate to acknowledge that the sub-formula property entails conservativeness because if o does not occur in a derivable sequent $\mathcal{S}$, then there is a cut-free derivation of $\mathcal{S}$ where neither $\circ$ nor its operational rules occur. Therefore, we have to show that each one of $\mathbf{L K}, \mathbf{L J}, \mathbf{L D J}$, and $\mathbf{L K R}$ admits cut-elimination. For LK and LJ this has been proved in [14] and for LDJ this has been proved in 40. Finally, we can prove cut-elimination in LKR by modifying the procedure given in [14 along the following lines: we consider directly Cut instead of Gentzen's Mix (to avoid the need of weakenings in the

\footnotetext{
17 Also for $\wedge, \vee$, and $\exists$ we can use the same derivations in each one of the calculi we are considering. For $\neg$ we have to invert the order of application of right and left rules in moving from $L J$ to $L D J$ (or vice versa).

18 Where, for any $y, A[y / x]$ is a sub-formula of $\forall x A$ and of $\exists x A$.
} 
transformed derivations) and, if contraction is principal in one of the premisses of Cut, we proceed as in [26]. To illustrate, we might transform

$$
\frac{\frac{A, \Pi \vdash \Sigma, B}{\Pi \vdash \Sigma, A \supset B} R \supset \frac{\Gamma_{1} \vdash \Delta_{1}, A \quad B, \Gamma_{2} \vdash \Delta_{2}}{A \supset B, \Gamma_{1}, \Gamma_{2} \vdash \Delta_{1}, \Delta_{2}}}{L \supset} \text { Cut }
$$

into

$$
\frac{\Gamma_{1} \vdash \Delta_{1}, A \quad A, \Pi \vdash \Sigma, B}{\frac{\Pi, \Gamma_{1} \vdash \Sigma, \Delta_{1}, B}{\Pi, \Gamma_{1}, \Gamma_{2} \vdash \Sigma, \Delta_{1}, \Delta_{2}} \quad B, \Gamma_{2} \vdash \Delta_{2}} \text { Cut }
$$

We have taken uniqueness and conservativity as a criterion of admissiblity for the operators of a calculus, and indeed for logics themselves, and we have shown that each one of $\mathbf{L K}, \mathbf{L J}$, LDJ, and LKR satisfies them. This means that each one of these calculi determines a valid - i.e., harmonious derivability relation, and that its derivable sequents can be read as in $(\star \star)$ and determine an admissible logic. We thus claim that the present form of proof-theoretic pluralism includes classical, intuitionistic, dual-intuitionistic, and some relevance logic as desired. Moreover, these calculi share the same set of operational rules; therefore the logical operators have the same meaning in all these admissible logics.

\section{Relevance logics within proof-theoretic pluralism}

If we consider $\mathbf{L K R}$ from the perspective of the given proof-theoretic account of validity, we don't have the problem that the rules of weakening should be valid. This problem was a consequence of the model-theoretic account of validity, but it disappears once we move to a proof-theoretic one based on Belnap's explication of harmony. The reading of sequents proposed in $(\star \star)$ does not necessarily entail that if the cases in $\Delta$ follow from the assumptions in $\Gamma$ then the cases in $\Delta, B$ follow from the assumptions in $\Gamma, A$. It does so only when considering formal explications of following from that are closed under the rules of weakening. When, e.g., we are reasoning within Euclidean geometry, we employ a notion of following from that is closed under weakening since we are in a paradigmatic classical context. But, the notion of following from is not closed under weakening in relevant contexts. As an example, let's consider the context of evaluating philosophical arguments. If a referee rejects a paper we have written because the only argument for our main conclusion $B$ is an instance of ex falso quodlibet ${ }^{19}$ we shouldn't respond: but $B$ follows from $A$ and $\neg A$ ! Another related example is the following one: suppose our main argument for $B$ is an argument from the assumptions $A$ and $C$. If someone

19 As it is shown in $\sqrt{2}$, in sequent calculi based on the rules in Tables 1 and 2 the $e x$ falso quodlibet $(\perp \supset A)$ is a consequence of the rule of right weakening. 
shows us that $B$ follows from $A$ alone, the natural answer would be to say that we were wrong in believing that $B$ followed from $A$ and $C{ }^{20}$

Notice that among the admissible logics there is not only the relevance logic LKR, but also the relevance logic LJR (LDJR, respectively) obtained by removing weakenings and by considering single-conclusion (single-premiss, respectively) sequents.

Now it is time to dispel a possible worry about our proposal. Kouri [19] asks for criteria delimiting the set of admissible logics that are obtainable by imposing structural restrictions on top of the operational rules given in Table 1. whereas harmony, which is our admissibility criterion, is normally meant to determine which sets of operational rules define a meaningful operator. Thus, someone might believe that our proposal doesn't provide what she is after. But notice that we have opted for a notion of harmony which holds if the rules satisfy uniqueness and conservativity over an antecedently given derivability relation that we have assumed to be reflexive and transitive. This immediately rules out the calculi that are obtained by imposing the structural restriction of having no formula to the left or to the right of $\vdash$ : these restrictions wouldn't give us a reflexive relation. Hence, harmony, when coupled with the assumption that the derivability relation is reflexive, rules out the structural restrictions that Kouri wants to exclude. Moreover, as it is convincingly argued in [6. p. 739], the following co-determination thesis holds in proof-theoretic pluralism:

the structure of the derivability relation is as much an effect of the operational rules as it is the effect of direct structural stipulations.

As a consequence, even if harmony delimits directly only which sets of operational rules are admissible, it also indirectly delimits the set of admissible structural restrictions. For example, suppose we start from a calculus that is like the one in Tables 1 and 2 save for the left operational rule for implication which is replaced by:

$$
\frac{\Gamma \vdash \Delta, A \quad B, \Gamma \vdash \Delta}{A \supset B, \Gamma \vdash \Delta} L \supset^{\prime}
$$

In this case, no relevance logic would be admissible. The problem is that we wouldn't be able to prove the uniqueness of implication as in (3), but we would need something like

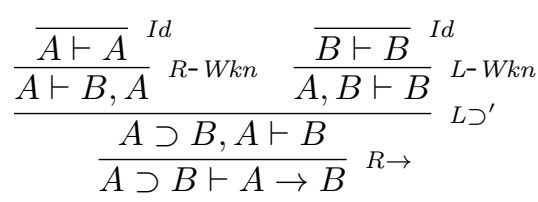

where we cannot eliminate the instances of the rules of weakening. As a consequence, no relevance logic would be harmonious with the left rule for implication in (5).

\footnotetext{
20 These responses require an abandonment of the universal applicability of logic. As we have pointed out at the end of Section 3 this is the natural choice to make within our framework.
} 
Finally, in the present context Belnap's notion of harmony is strongly bounded to the reflexivity and transitivity of the derivability relation. There are two reasons for this fact. First, the rule $I d$ that expresses reflexivity has been used in proving the uniqueness of the operators and we don't know whether it would be possible to prove uniqueness without it. Hence, regardless of whether we start by assuming reflexivity as one of the distinctive features of the derivability relation or not, we conjecture that it wouldn't have been possible to show the admissibility of any non-reflexive logic. If this conjecture is correct, then harmony rules out the structural restriction that Kouri wants to exclude independently of our assumption that the derivability relation is reflexive. Second, we have proved conservativity by relying on cut-elimination, hence the calculi we have considered are closed under the rule of Cut regardless of whether this rule is primitive or not. Given that Cut expresses the transitivity of the derivability relation, we have that in all the admissible logics that are obtained by the rules in Tables 1 and 2 the derivability relation must be transitive. We conclude that harmony provides a criterion for delimiting the sets of structural restrictions that determine admissible logics.

We are now in a position to lay out our proposal of an harmony-based proof-theoretic MILP in full detail. We have started by assuming that a derivability relation $\vdash$, which is the formal representation of the notion of following from, is a reflexive and transitive relation between pairs of multisets of firstorder formulas. Sequent calculi have been used to determine logics, where formally a logic can be identified with the extension of the derivability relation in a calculus. We have also assumed that a logic is admissible whenever its operators satisfy conservativity and uniqueness with respect to $\vdash$, and that the meaning of each logical operator $\circ$ is completely determined by its left and right operational rules in sequent calculi. We have thus obtained a form of proof-theoretic MILP where a family of admissible logics sharing the same operators is obtained by changing the structural elements of deduction-i.e., by changing the structural rules of inference and/or the definition of how sequents are composed.

Starting from the rules given in Tables 1 and 2, we have considered a family of admissible logics that includes classical logic $\mathbf{L K}$, intuitionistic logic $\mathbf{L J}$, dual-intuitionistic logics LDJ, as well as their relevant versions LKR, LJR, and LDJR. In each of these logics the operators $\neg, \wedge, \vee, \supset, \forall$, and $\exists$ have the same meaning since they share the same meaning-conferring operational rules. Depending on the situation it might be appropriate to reason according to one or another of these (admissible) logics. Notice that the set of admissible logics that we have shown to be in this family is not exhaustive since, e.g., we have not considered logics without contraction ${ }^{21}$ Moreover, we have not claimed that for each logic in this set there is some situation where we have

\footnotetext{
21 Notice that we cannot have non-reflexive or non-transitive admissible logics within this family because it is based on a reflexive and transitive derivability relation. Hence, one might want to distinguish between the structural rules that are essential ingredients of the admissible logics - in that they express distinctive characteristic of the derivability relation - and those that can be modified to obtain the different admissible logics.
} 
to reason according to it. In particular, we maintain that at least each of classical, intuitionistic and some relevance logic is the appropriate canon of reasoning in some situation. We have illustrated this by considering the case of reasoning within classical real analysis and Euclidean geometry where we have to use $\mathbf{L K}$, that of reasoning within constructive analysis where we have to use $\mathbf{L} \mathbf{J}$, and that of evaluating philosophical arguments where we have to use a relevance logic such as LKR. We also conjecture that some other logic considered here might be appropriate in some situation, e.g., it might be that sometimes we have to reason both constructively and relevantly and thus to use LJR. Nevertheless, we don't have in mind any particular situation where we have to reason according to the single-premiss calculi LDJ and LDJR.

\section{Families of harmonious logics}

Let us briefly take stock. So far we have been focusing exclusively on the task of showing how to extend the basic framework of proof-theoretic pluralism by developing a particular family of logics within proof-theoretic MILP which encompasses all of classical, intuitionistic, dual-intuitionistic, and some relevance logics - i.e., we have provided a proof-theoretic alternative to the model-theoretic MILP presented in [1]. Having accomplished that task, in this section we would like to briefly explore what we think is a promising way of expanding the proof-theoretic pluralist framework beyond MILP 22

An important issue has remained in the background until now: the possibility of changing logic by changing the operational rules and by starting with a notion of derivability differing from the one we have chosen. This possibility is compatible with a proof-theoretic pluralism based on Belnap's global harmony. In principle, modulo certain assumptions on the derivability relation, it is possible to start from any well-behaved combination of operational rules and structural elements and then determine which logics are admissible with respect to that particular choice. By 'well-behaved' here we simply mean that that combination must be not only congruent with the assumptions on the derivability relation but also with uniqueness and conservativity. Different choices might make different set of logics admissible: each choice generates a family of admissible logics. Within each family the logical operators have the same meaning, but the meaning of a logical symbol might vary in moving from one family to another. We thus have a two-level proof-theoretic pluralism: (i) the first level, MILP is obtained by varying the structural elements within a given family while keeping the same operational rules; (ii) the second level, MVLP is obtained by varying the set of distinctive properties of the derivability relation and/or the operational rules themselves. It is important to stress that both MILP and MVLP are constrained by the same principle of harmony. In this respect, we take harmony to give a common core for all the admissible logics, thus making our proposal a from of MVLP that, arguably, is less liberal than a fully Carnapian one.

22 Thanks are due to an anonymous referee for helping us clarifying this issue. 
Table 3 Operational rules for intensional conjunction $\otimes$ and disjunction $\oplus$

\begin{tabular}{ll}
\hline$\frac{A, B, \Gamma \vdash \Delta}{A \otimes B, \Gamma \vdash \Delta} L \otimes$ & $\frac{\Gamma \vdash \Delta, A \quad \Pi \vdash \Sigma, B}{\Gamma, \Pi \vdash \Delta, \Sigma, A \otimes B} R \otimes$ \\
$\frac{A, \Gamma \vdash \Delta \quad B, \Pi \vdash \Sigma}{A \oplus B, \Gamma, \Pi \vdash \Delta, \Sigma} L \oplus$ & $\frac{\Gamma \vdash \Delta, A, B}{\Gamma \vdash \Delta, A \oplus B} R \oplus$ \\
\hline
\end{tabular}

We now illustrate how MVLP works within our two-level pluralism by outlining two other families of admissible logics that are used in discussing the semantic paradoxes ${ }^{23}$ First, there is a family of admissible logics that are used in substructural approaches to the semantic paradoxes. Let us consider a reflexive and, possibly, non-transitive derivability relation between pairs of multisets. The logics in this family are generated by considerning the structural rules in Table 2 and the operational rules for negation and implication given in Table 1 and the rules for intensional conjunction and disjunction that are given in Table 3 . Among the admissible - i.e., harmonious - logics in this family there are a non-contractive logic and a non-transitive logic that are used in substructural approaches to the semantic paradoxes, see [31. Moreover, within this family there is a calculus for classical logic that differs from $\mathbf{L K}$, as well as calculi for relevance logics that are not coextensional with the one we have considered.

Next, we move to another interesting family where, among the admissible logics, there are calculi for the logics FDE, LP, and K3 that can be used in many-valued approaches to the semantic paradoxes. This family is obtained by starting from a four-sided reflexive and transitive derivability relation. On top of this derivability relation we take as operational rules a four-sided version of the rules for $\otimes$ and $\oplus$ given in Table 3 as well as four shifting rules for negation - where a shifting rule is a rule moving formulas form one side of the derivability relation to another side - and, as structural rules, we consider the (four-sided version of the) rules of weakening, contraction, $I d$, and Cut, and four shifting rules that allows to move formulas between the first two and the last two multisets of the derivability relation. Depending on which of the shifting structural rules we allow ourselves to use we get either FDE, or LP, or K3, or a calculus for classical logic, see 32 for the details.

As we have already said, within each of these families the logical operators have the same meaning, but this is not so when we move from one family to another. We thus obtain a two-level form of logical pluralism in which, to recap, the first level is the MILP given by the set of admissible logics obtained by keeping the same operational rules and by changing the structural elements of deduction, while the second level is a MVLP that is obtained by changing the operational rules and, possibly, the basic definition of the derivability relation. This MVLP is related to Carnap's one [5]. But it differs

\footnotetext{
23 For the sake of brevity, we limit our considerations to the propositional fragment of the language. Moreover, we don't consider the truth predicate as part of the logical language (so that we don't have to check whether its rules are harmonious or not).
} 
from it in that whereas for Carnap 'everyone is at liberty to build his own logic' [5, §17], here this holds only as far as one can show that one's logic is harmonious. Recall that, following Belnap, harmony is explicated in terms of uniqueness and conservativity. Hence, in assuming this explication of harmony we take both uniqueness and conservativity to be indispensable features that any logic should satisfy in order to be admissible. In this respect, the only constraint that is imposed on the choice of a derivability relation and a set of operational and structural rules is given by the need to prove both uniqueness and conservativity. Given that, to the best of our knowledge, no proof of uniqueness has been given without $I d$ we have conjectured that the derivability relation cannot be non-reflexive if we want to have some logic admissible ${ }^{24}$ Notice that this does not mean that we take reflexivity as a core feature of the derivability relation as such but only that, given the explication of harmony that we have assumed, within each family we have that one of the distinctive characteristics of the derivability relation will be reflexivity. In this sense, our two-level proof-theoretic pluralism can still be said to be harmony-based. In fact, harmony constraints not only the set of logics that are admissible within a given family but also the set of families that we can consider - e.g., it might exclude all families that are based on a non-reflexive derivability relation. This makes our framework broadly Carnapian in that it is highly tolerant but still somehow constrained. Alternatively, one could develop a framework where we have both an internal constrain on admissible logics within a family given by harmony as well as an external constrain on 'admissible' families given by imposing core features that every derivability relation must satisfy. This would be an alternative two-level framework which would score as less tolerant, and thus less Carnapian, than the one we have sketched in this section 25 This is not the place to discuss at length the MVLP-level of our two-level framework given that we are focusing on MILP; nevertheless it is interesting to notice that the two forms of pluralism can happily coexist in our proof-theoretic approach.

\section{Proof-theoretic pluralism meets Necessity}

After the brief discussion of MVLP and the two-level framework, let's get back to our main focus - namely, MILP and the family of admissible logics containing LK, LJ, LDJ, and LKR. Beall and Restall in [1, pp. 14-23] introduce and discuss three features - Necessity, Normativity, and Formality - that are traditionally taken to be distinctive of deductive logical systems, as opposed e.g. to inductive logical systems. For this reason, Beall and Restall take them to be

\footnotetext{
24 The only logic inhabiting a non-reflexive family with no operational rules would trivially satisfy both conservativity and uniqueness. As it is usual in debates about logical pluralism, we are interested only in logics containing some operators and in the relation between the operators of the different logics. As a consequence, we will consider only families of logics containing some operational rules where rule $I d$, and hence reflexivity, is needed to prove uniqueness. Thanks are due to an anonymous referee on this point.

25 We would like to thank an anonymous referee for pressing us to make clear this distinction.
} 
constitutive features of logic in that they function as the admissibility criteria for a logic. In this and the next two sections, we will briefly describe each of these features for then showing that our proof-theoretic MILP satisfies them. However, differently from Beall and Restall, we don't take these three features to function as admissibility criteria for a logic given that we take harmony to play that role in our framework. We consider them as theoretical desiderata in the following sense: by showing that all the admissible - i.e., harmonious logics satisfy them we have an argument for claiming that all of these logics are materially adequate. In this respect, satisfaction of these three features can be taken as abductive evidence for the adequacy of our proposal.

Let's begin with Necessity. The intuitive idea behind this feature is that there is a necessary connection between a statement and its logical consequences. Given the predominance of model-theoretic treatments of modalities, the necessity feature finds its natural home in a model-theoretic setting. Within this trend, Beall and Restall [1, pp. 14-15] take Necessity in a framework that allows for multiple conclusions to amount to the idea that the truth of all premises necessitates the truth of some of the conclusions where 'necessitates' here is quite naturally understood along the line of the traditional characterisation in terms of truth in all models (or possible worlds).

If we move away from a model-theoretic setting and endorse a prooftheoretic one, it is not immediate to see how to render the necessity feature ${ }^{26}$ Since for the purpose of this paper we won't have the space to embark on the project of providing a full account of a proof-theoretically friendly version of the necessity feature, we will rest content if we can at least offer an outline of how such an account might work. With this modest aim in hand, we take it that a promising starting point is given by a passage from Aristotle's Prior Analytics where he writes:

A deduction is a discourse in which, certain things having been supposed, something different from the things supposed results of necessity because these things are so. By "because these things are so", I mean "resulting through them" and by "resulting through them", I mean "needing no further term from outside in order for the necessity to come about". [An. Pr. I, 2, 24b 20-25]

Leaving exegetical details aside, it is interesting to note that in providing an informal gloss of the necessity feature of logical consequence, Beall and Restall themselves seem to have in mind something which echoes, at least at first glance, the thought expressed by Aristotle's passage. They write:

The fact that logical consequence is necessary means that logical consequence applies under any conditions whatsoever [...] The applicability

26 We can give a model-theoretic consequence relation that is adequate for each logic we are considering. For LK, LJ, and LDJ models can be defined as in 30; for LKR, LJR, and LDJR we can use the uni-residuated lattice-ordered grupoids considered in [8]. These model-theoretic consequence relations would show that the admissible logics in our approach satisfy the model-theoretic notion of necessity. 
of logic is not a contingent matter; it works come what may, whatever hypotheses we care to entertain. [1, pp. 15-16]

We can capture the gist of what this informal characterisation of necessity have in common by saying that according to the necessity feature, logical consequence is that kind of relation between premises and conclusion(s) that occurs solely in virtue of characteristics that are intrinsic to the deductive system in use and thus entirely independent of further, extra-logical, hypotheses that we may entertain and which are external to the system.

If we endorse this qualification of the informal characterisation of Necessity, it can be easily seen that the proof-theoretic pluralism developed in this paper fully accounts for it. Given a certain context of deducibility, and thus a range of admissible logics, we have that an argument, if valid in an admissible logic, it remains valid in that logic under any conditions whatsoever, regardless of any extra-logical factors. In this sense, the fact that an argument is valid is not a contingent matter - although it might be a relative matter, i.e. relative to which of the admissible logics we are using ${ }^{27}$ It is interesting to point out at this point that there seems to be a very tight connection between the notion of formality which we take to be at the core of the formality feature and the characterisation of Necessity just sketched. To illustrate this point, we now turn to a discussion of Formality.

\section{Proof-theoretic pluralism meets Formality}

With respect to Formality, Beall and Restall [1, p. 21], following MacFarlane 20], distinguish between three ways in which it can be characterised: (i) logic is formal in the sense that it provides constitutive norms for thought as such; (ii) logic is formal in that it is indifferent to the particular identities of objects; (iii) logic is formal in the sense that it abstracts entirely from the semantic content of thought. Beall and Restall do not commit to one specific characterisation of Formality, and when they check whether each of the admissible logics in their model-theoretic account satisfies this core feature, they briefly discuss each of these characterisations.

Without going into the many complex issues that these three characterisation of Formality and their relationships give rise to ${ }^{28}$ we take it that the core of the matter for the purposes of assessing whether our proof-theoretical pluralism is a genuine form of pluralism according to the parameters set in [1], resides in the thought that the validity of an argument solely depends on its logical form - or, its schematic features - and thus it abstracts entirely from the specific semantic content of its premises and conclusions. In other words, we take the characterisation of Formality expressed by (iii) above to provide

\footnotetext{
27 Another strategy that seems viable to us is to adopt what Bueno and Shalkowski 4 call modalism, namely the view that some modality, and in particular the modality involved in the necessity feature, is treated as primitive and adapt it to a proof-theoretic pluralist setting.

28 For a detailed discussion of these three characterisation of Formality, see [20].
} 
the starting point for understanding this feature within the context of our project. In fact, once we take this characterisation of Formality on board and we supplement it with a proof-theoretic criterion for logicality (see below), we can easily account for the thought that logic is purely formal.

Let's now see why our proof-theoretic pluralism meets Formality, understood along the line of characterisation (iii) above. We start by reflecting on the fact that it is immediate to see that in each of the logics which are admissible in the proof-theoretic framework developed in this paper, valid arguments are schematic: they all concern argument forms and they don't speak at first of individual concrete arguments. However, as Beall and Restall [1, p. 20] point out, the mere fact that an argument is schematic might not be sufficient for an adequate characterisation of Formality since there are argument forms i.e. purely schematic arguments - which do not seem to be logical argument - i.e. deductively valid arguments. To illustrate this point with a couple of examples taken from Beall and Restall [1, p. 20], let's consider the following two arguments that are schematic and necessarily truth preserving but are not, intuitively, deductively valid and thus logical arguments: (i) $x$ is larger than $y, y$ is larger than $z$; so $x$ is larger than $z$; (ii) $x$ is red; therefore $x$ is coloured. Both argumentative forms seem to be perfectly fine but they work in virtue of something other than purely logical reasons - i.e. reasons that have to do purely with the connectives and quantifiers of predicate logic. In fact, the first example relies on the very plausible principle that the relation larger than is transitive, while the second example hinges on the analytic connection between colour concepts.

Thus, in order to show that all the logics which are admissible within our proof-theoretic pluralist framework are (iii)-Formal more is needed than the mere idea that these logics are schematic. Specifically, what is needed is a criterion for distinguishing between logical and non-logical vocabulary. If we adopt the proof-theoretic criterion of logicality introduced by Došen [9, p. 366], according to which an operator is logical if it can be ultimately analysed in structural terms ${ }^{29}$ we have all we need to characterise a notion of (iii)Formality which is fully adequate and satisfies the proof-theoretic pluralism developed here. Under Došen's analysis of logicality, an operator is logical if it represents some structural element of deduction, and, starting from the rules in Table 1 , it is possible to show that each one of $\neg, \vee, \wedge, \supset$ and, extending to first order logic, each one of $\forall, \exists$, represents some structural elements of deduction, see [9] for the details. Thus, with all this in hand, valid arguments within our proof-theoretic framework are formal.

As for characterisations (i) and (ii), we get them for free. Characterisation (i) immediately follows from the fact that proof-theoretical pluralism satisfies Normativity and Formality as we understand them (see below, section 10). Characterisation (ii) is a direct consequence of the fact that proof-

\footnotetext{
29 As argued in [16], Došen's proof-theoretic criterion for logicality is related to the notion of harmony, but it is independent from it. Hence, it makes sense to supplement a prooftheoretic pluralism based on harmony with this criterion of logicality.
} 
theoretic pluralism satisfies Formality, once properly supplemented with a proof-theoretic criterion for logicality.

\section{Proof-theoretic pluralism meets Normativity}

Normativity is the thesis that there is a normative link between logic and reasoning in (at least) the sense that if a subject accepts all of the premises of a valid argument while at the same time refraining from accepting each of its conclusions, she is doing something incorrect. The way in which Beall and Restall characterise Normativity is the following: 'In an important sense, if an argument is valid, then you somehow go wrong if you accept the premises but reject the conclusion' [1, p.16]. In this minimal sense, it seems quite uncontroversial to say that logic normatively constrains reasoning (or, at least, some aspects of it). However, how exactly to understand this idea of incorrectness or wrong doing and what is the precise nature of the normative link between logic and reasoning is a matter of discussion among philosophers ${ }^{30}$

Each of the logics that are admissible within the proof-theoretic pluralism developed in this paper respect Normativity, at least in the minimal sense we have just sketched. This is because, in general, proof-theoretic approaches to logic give the meaning of the logical vocabulary in terms of harmonious rules and thus are easily amenable to normative characterisations. For instance, one natural interpretation of sequent calculi given by Restall [29] explicitly takes the calculi as supplying a normative constraint on acts of assertion and denial: if a sequent $\Gamma \vdash \Delta$ is valid, then an agent cannot rationally assert all members of $\Gamma$ while, at the same time, denying all members of $\Delta$. Rules for operators in such calculi show how to extend those constraints to assertions or denials of complex expressions. If we endorse this bilateral interpretation of the calculi and model the normative constraint along the lines specified in Normativity, we have everything we need to ensure that all the admissible logics within our proof-theoretic pluralism respect Normativity. That said, the exact respects under which the notion of logical consequence is normative for deductive reasoning is a rather complex issue, as recent discussions in the philosophy of logic show. A variety of fundamental questions are currently investigated which concern the structure of the so-called 'bridge principles' (see, e.g., 21] and 37]) - i.e., principles bridging facts about logic with norms governing reasoning - and the source and nature of the normative notions involved in these principles (see [37]). For the purpose of this paper, we cannot go into the details of this intricate debate. We thus leave the interesting question of how exactly the normative constraint should be understood and modelled within our proof-theoretic pluralism for future work.

However, before concluding, we would like to mention an advantage that the version of proof-theoretic pluralism sketched in this paper has over the model-theoretic pluralism defended in [1. As argued in [13, model-theoretic

\footnotetext{
30 For a critical survey of some of the main issues in connection with the normative status of logic we invite the reader to refer to [37.
} 
pluralism suffers from a variety of challenges concerning the normative role that the notion of 'follows from' plays in connection with (deductive) reasoning. The source of these challenges resides in the fact that they take the English expression 'follows from' to be semantically indeterminate - they explicitly draw a parallel with the vagueness debate [1, p. 27]. More precisely, three different precisifications can be given of the notion of validity, depending on how the notion of 'case' in the Generalised Tarski's Thesis is specified.

To have a better grip on these challenges, let us take a step back and briefly explain the framework elaborated in [1]. According to Tarski, logical consequence should be understood in terms of necessary truth preservation [38, p. 411], which, in turn, can be sharpened model-theoretically as follows: a sentence $A$ follows logically from a set of sentences $\mathcal{S}$ just in case every model of $\mathcal{S}$ is a model of $A$ [38, p. 417]. Roughly, Tarski defined a model of a set of sentences $\mathcal{S}$ as a way of interpreting the sentences that would make them come out as true, by assigning semantic values of the appropriate kind to each type of non-logical expressions. Crucially, Tarski took these models to yield classical logic [38, p. 197] (see also [1, p. 39]). According to [1, Tarski's conception can be generalized in order to allow for notions other than Tarskian models and thus for different logics than classical logic. This is done by means of the following schematic definition of validity:

GENERALIZED TARSKIS THESIS (GTT): an $\operatorname{argument}^{\text {is }} \operatorname{valid}_{x}$ if and only if, in every case $_{x}$ in which the premises are true, so is the conclusion.

The notion of a case $_{x}$ is intended to include among its instances not only Tarskian models, yielding classical logic, but also other notions such as constructions and situations that, respectively, give us intuitionistic logic and relevant logic. A plurality of consequence relations thus results from the variety of ways of understanding the notion of a case $x_{x}$ over which (GTT) quantifies. On that basis, different interpretations of how to understand the relation between the formal framework and the ordinary English expression 'follows from' can be given. If we take the textual evidence given in [1, pp. 27-29] at face value, the most plausible interpretation is to take Beall and Restall as claiming that the notion of logical consequence is semantically indeterminate in the sense that there are different but equally admissible ways of precisifying it. Regardless of which specific semantic characterisation of indeterminacy we provide - either a variety of underspecificationist models or a variety of overspecificationist models - arguments can be given to show that model-theoretic pluralism fails to offer an adequate account of the normative guidance that logic is taken to provide us within ordinary contexts of reasoning.

The version of proof-theoretic MILP developed in this paper is immune to this line of criticism. As we have seen above, once we are within a settled family of admissible logics the notion of derivability at its core has an invariant meaning and thus is not schematic in the sense of [1. In fact, it is not based on anything like the Generalised Tarski's Thesis and thus it is not subject to 
the kind of semantic indeterminacy of the expression 'follows from' that creates problems within the framework developed in [1. In this paper we haven't engaged with the issue of how to interpret semantically the expression 'follows from', and for reason of length we won't dig into this issue. However, it is clear that we are not forced to endorse the kind of semantic indeterminacy model at the core of the proposal in [1. We take it that, within each family of admissible logics, a viable semantic model for the English expression 'follows from' which is fully in consonance with the pluralist framework developed in this paper is a non-indexical-contextualist one. Assuming a standard Kaplanian model for handling context sensitivity, the thought would be that 'follows from' always denotes the same function from circumstances of evaluations to extensions. Thus, intentionally, 'follows from' has the same meaning in all contexts in which it occurs but its extension varies in relation to the specific context in which it is used - depending on whether we are reasoning within, e.g., a classical or an intuitionistic context ${ }^{31}$ In this respect, the proof-theoretic pluralism sketched here offers a clear advantage over the model-theoretic version developed in [1].

\section{Conclusion}

In this paper we have shown that if we supplement the basic framework of proof-theoretic pluralism with a proof-theoretic criterion of admissibility, such as Belnap's notion of harmony, we obtain all of classical, intuitionistic, and dual-intuitionistic, as well as some relevance logics, as admissible logics. Hence, harmony provides an admissibility criterion that is better behaved than the model-theoretic one considered in 19 - at least for anyone who wants some relevance logics among the admissible ones. Moreover, the adoption of harmony as an admissibility criterion is more in line with the spirit of proof-theoretic pluralism, and therefore it should be preferred to model-theoretic ones even by someone who is not moved by considerations about relevance. We have also argued that within an harmony-based proof-theoretic pluralism we have both a meaning-invariant (MILP) and a meaning-variant (MVLP) version of logical pluralism. Finally, we have considered Beall and Restall's 1 desiderata of Necessity, Normativity, and Formality, and we have argued that they are satisfied by the logics that are admissible in proof-theoretic MILP.

\section{References}

1. Beall, J. \& Restall, G., Logical Pluralism. Oxford University Press, Oxford (2006)

2. Beall, J. et al., Relevant restricted quantification, Journal of Philosophical Logic, 35(6), 587-598 (2006)

3. Belnap, N.D., Tonk, plonk and plink, Analysis, 22(6), 130-134 (1962)

\footnotetext{
31 For more details on how this proposal of the semantic of 'follows from' might work, we invite the reader to have a look at Chapters 4 and 5 of 33 .
} 
4. Bueno, O. \& Shalkowski, S., Modalism and logical pluralism, Mind, 118(470), 295-321 (2009)

5. Carnap, R., the Logical Syntax of the World. Routledge \& Kegan Paul Ltd., New York (1937)

6. Dicher, B., A proof-theoretic defence of meaning-invariant logical pluralism, Mind, 125(499), 727-757 (2016)

7. Dicher, B., Weak disharmony: Some lessons for proof-theoretic semantics, The Review of Symbolic Logic, 9(3), 583-602 (2016)

8. Došen, K., Sequent-systems and grupoids models, Studia Logica, 47, 353-386 (1988) and 48, 41-65 (1989)

9. Došen, K., Logical constants as punctuation marks, Notre Dame Journal of Formal Logic, 30(3), 362-381 (1989)

10. Dummett, M., Frege: Philosophy of Language. Harvard University Press, Harvard (1973)

11. Dummett, M., The Logical Basis of Metaphysics. Duckworth, London (1991)

12. Dunn, J.M. \& Restall, G., Relevance logic, Handbook of Philosophical Logic, Vol. 6 (2 ${ }^{\text {nd }}$ ed.), 1-128. Kluwer, Dordrecht (2002)

13. Ferrari, F. \& Moruzzi, S., Logical pluralism, indeterminacy and the normativity of logic, Inquiry, https://doi.org/10.1080/0020174X.2017.1393198 (2017)

14. Gentzen, G., Untersuchungen über das logische schliessen, Mathematische Zeitschrift, 39, 176-210 and 405-431 (1934-35)

15. Gratzl, N. \& Orlandelli, E., Double-line harmony in a sequent setting, Logica Yearbook 2016, 157-171. College Publications, London (2017)

16. Gratzl, N. \& Orlandelli, E., Logicality, double-line rules, and modalities, Studia Logica, 107(1), 85-107 (2019)

17. Haack, S., Deviant Logic. C ambridge University Press, London (1974)

18. Hjortland, O.T., Logical pluralism, meaning-variance, and verbal disputes, Australasian Journal of Philosophy, 91(2), 355-373 (2013)

19. Kouri, T., Restall's proof-theoretic pluralism and relevance logic, Erkenntnis, 81, 1243 $1252(2016)$

20. MacFarlane, J., What does it mean to say that logic is formal?, PhD thesis, University of Pittsburgh (2000)

21. MacFarlane, J., In what sense (if any) is logic normative for thought?, Unpublished manuscript, http://johnmacfarlane.net/normativity_of_logic.pdf (2004)

22. Moriconi, E. \& Tesconi, L., On inversion principles, History and Philosophy of Logic, $29(2), 103-113(2008)$

23. Naibo, A. \& Petrolo, M., Are uniqueness and deducibility of identicals the same?, Theoria, 81, 143-181 (2015)

24. Negri, S. \& von Plato, J., Structural Proof Theory. Cambridge University Press, Cambridge (2001)

25. Paoli, F., Quine and Slater on paraconsistency and deviance, Journal of Philosophical Logic, 32(5), 531-548 (2003)

26. von Plato, J., A proof of Gentzen's Hauptsatz without multicut, Archive for Mathematical Logic, 40(1), 9-18 (2001)

27. Prior, A.N., The runabout inference ticket, Analysis, 21(2), 38-39 (1960)

28. Read, S., Harmony and autonomy in classical logic, Journal of Philosophical Logic, 29, 123-154 (2000)

29. Restall, G., Multiple conclusions, 12 ${ }^{\text {th }}$ International Congress on Logic, Methodology and Philosophy of Science, 189-205. KCL Publications, London (2005)

30. Restall, G., Pluralism and proofs, Erkenntnis, 79, 279-291 (2014)

31. Ripley, D., Comparing substructural theories of truth, Ergo, 2(13), 299-328 (2015)

32. Shapiro, L., LP, K3, and FDE as substructural logics, Logica Yearbook 2016, 257-272.

College Publications, London (2017)

33. Shapiro, S., Varieties of Logic. Oxford University Press, Oxford (2014)

34. Steinberger, F., Tennant on multiple conclusions, Logique et Analyse, 51(201), 49-55 (2008)

35. Steinberger, F., What harmony could and could not be, Australasian journal of Philosophy, 89, 617-639 (2011)

36. Steinberger, F., Why conclusions should remain single, Journal of Philosophycal Logic, 40, 333-355 (2011) 
37. Steinberger, F., The normative status of logic, The Stanford Encyclopedia of Philosophy (Spring 2017 Edition), https://plato.stanford.edu/archives/spr2017/entries/logicnormative/ (2017)

38. Tarski, A., Logic, Semantics, Metamathematics. Clarendon Press, Oxford (1956)

39. Tennant, N., The Taming of the True. Oxford University Press, Oxford (1997)

40. Urbas, I., Dual-intuitionistic logic, Notre Dame Journal of Formal Logic, 37(3), 440-451 (1996) 